Journal of Computer Science 7 (2): 270-278, 2011

ISSN 1549-3636

(C) 2011 Science Publications

\title{
Mobility Management for Seamless Information Flow in a Heterogeneous Networks
}

\author{
${ }^{1}$ Adiline Macriga and ${ }^{2}$ Anandhakumar \\ ${ }^{1}$ Department of Information and Communication, \\ ${ }^{2}$ Department of Information Technology, \\ MIT Campus, Anna University Chennai, 600025, Chennai
}

\begin{abstract}
Problem statement: In the heterogeneous networks, generally the entire networks operating on the Radio Frequencies under wireless mode available for communication are brought under a single head based on their common operating procedures and functions. As a common mode the basic functions of the networks are requesting, tracing, sending, acknowledging, authenticating, authorizing, receiving, updating, forwarding, controlling, registering, managing. As the radio frequency is considered the signal strength, maximum coverage, power level are taken as the major factor. Approach: Todays cellular network has a wide coverage area and also a good signal strength and as the WiMax is considered the data transmission rate and the volume of information communicated is high. As we consider the Adhoc Network the path tracing and the nearby node identification is faster and there is no need for a standard architecture. As the major of the different networks are combined with certain QoS without disturbing the existing infrastructure a fast seamless flow of voluminous information in real time application can be achieved. As the data transmission is considered the major part is affected based on the initiation \& delay such as authentication delay, handover delay, session initiation, activation which is being carried out only after the control had reached the service point. Results: In the proposed study the transmission of information is purely controlled by categorizing the type of information that is being transmitted such as the information ready for transmission is online or offline. Coming to on line either voice communication, Multimedia communication or real-time information transmission. Considering the offline the information is stored file or images. Among the above one the real time video transmission is considered as the major application because delay cannot be permitted and one more thing the mobility should be managed without any break in the transmission path. The rapidly growing demand for "anywhere, anytime" high-speed access to IP-based services is becoming one of the major challenges for mobile networks. Conclusion: As the demand for mobility increases, mobile terminals need to roam freely across heterogeneous networks, posing the challenge of network integration into an All-IP ubiquitous access platform.
\end{abstract}

Key words: Location tracking, location management, mobility management, heterogeneous networks, seamless mobility, Vehicular Area Network (VAN), seamless flow, ad hoc network, Session Management Protocol (SMP)

\section{INTRODUCTION}

Today's communication technology becomes outdated for tomorrows requirement. The growth of the communication industry is tremendous and unimaginable. There are different modes like "wired, wireless, adhoc, mobile, supporting the growth of the communication industry but with all certain limits. Now, it is time to emerge into the world of mobility where the wireless communication plays a vital role, where it is necessary to satisfy the requirements of the modern world. A world without a mobile phone is unimaginable. It has taken people to a different world. Now it is the time for providing services in an uninterrupted way. In medical industry a millisecond delay in transfer of information may lead to a loss of life. So the technology has to be updated day by day to meet the needs of the various industries. As the communication industry is considered it is one of the challenging one for the researchers. Considering the infrastructure of the existing communication industry a huge amount has been deployed by different service

Corresponding Author: T. Adiline Macriga, Department of Information and Communication, MIT Campus, Anna University Chennai, 600025, Chennai 
providers to satisfy their customer needs. It is now a challenge to provide the flow of information seamlessly without re-modifying the existing infrastructure. The most challenging one is to provide mobility management for real time communication services. This study mainly concentrates on framing a path in advance between the source and the destination based on the nature of the data being communicated in this case mobile video conferencing. This study discusses the challenges involved in the mobility management between the source and the destination and handoff technique. In heterogeneous wireless networks, traditionally the network is initiated with any request and immediately the type of request is identified as voice, data, image, data and image, motion pictures, live information, online videos, store and forward. Based on the type of information projected the network is chosen as if the transmission involves voice, voice and data, data and images the cellular network is chosen and if the transmission voluminous data and image transmission and the network coverage is short, the wifi or WLAN network is chosen. If the transmission involves live videos with emergency information an open connection between the transmitter and the receiver is proposed. As an example the following situation is considered. In case of emergency situation such as natural calamities and disaster-which require immediate recovery and attention by identifying the spot and also requires emergency medical help, where it is not possible to make the medical experts present for helping hand in person, a virtual communication media can be set up and the experts from remote areas can bring closer and the condition of the patient can also viewed by the medical experts so that they can provide a good medical treatment with the available practitioners. So that the invaluable life can be saved. This is the major purpose for choosing the research topic. Considering the handoff it is mainly classified as: horizontal handoff and vertical handoff. A horizontal handoff is made between different access points within the same link-layer technology such as when transferring a connection from one Base Station to another or from one Access Point to another. A vertical handoff is a handoff between access networks with different link-layer technologies, which will involve the transfer of a connection between a Base Station and an Access Point. Seamless and efficient Vertical Handoff between different access technologies is an essential and challenging problem in the development toward the next-generation wireless networks (Yu and Krishnamurthy, 2007; Zhu and Leung, 2007). Internally as the handoff process is considered it can be further carried out using the following main steps: system discovery, handoff decision and handoff execution (ParveenSultana et al., 2011). During the system discovery phase, mobile terminals equipped with multiple interfaces have to determine which networks can be used and the services available in each network. During the handoff decision phase, the mobile device determines which network it should connect to. During the handoff execution phase, connections need to be rerouted from the existing network to the new network in a seamless manner. There are three strategies for handoff decision mechanisms: mobile-controlled handoff, network-controlled handoff and mobileassisted handoff (Ma et al., 2007). Mobile Controlled Handoff is used in IEEE 802.11 WLAN networks, where a Mobile Host continuously monitors the signal of an Access Point and initiates the handoff procedure. Network Controlled Handoff is used in cellular voice networks where the decision mechanism of handoff control is located in a network entity. Mobile Assisted Handoff has been widely adopted in the current WWANs such as GPRS, where the mobile host measures the signal of surrounding base stations and the network then employs this information and decides whether or not to trigger handoff (Imre, 2007; Rodriguez-Dagnino and Takagi, 2007; Quintero and Frutos, 2009). The handoff algorithms (Imre, 2007) considered are based on the threshold comparison of one or more metrics and dynamic programming/artificial intelligent techniques applied to improve the accuracy of the handoff procedure. The most common metrics are received signal strength, carrier-to-interference ratio, signal-to-interference ratio and bit error rate (Holis and Pechac, 2008). In heterogeneous wireless networks, even though the functionalities of access networks are different, all the networks use a specific signal beacon or reference channel with a constant transmit power to enable received signal strength measurements. Thus, it is very natural and reasonable for vertical handoff algorithms to use received signal strength as the basic criterion for handoff decisions (Ma et al., 2007; Roberts et al., 2007).

The rest of the study is organized as follows: Section 2 provides the survey of existing technologies. Section 3 provides the solution for mobility management. Section 4 presents the proposed method. Section 4.1 presents the results and related discussion. Section 5 discusses directions for future work and concludes this study.

\section{MATERIALS AND METHODS}

In the real-time service, the number of continuous beacon signals should be lower than that of the non- 
real-time service in order to reduce the handoff delay. More parameters may be employed to make more intelligent decisions. Tamilselvan and Shanmugam (2011) propose a bandwidth aware VHO technique which considers the residual bandwidth of a WLAN in addition to RSS as the criterion for handoff decisions. However, it relies on the QBSS load defined in the IEEE 802.11e Standard to estimate the residual bandwidth in the WLAN. In (Hassan et al. (2009), propose a method for defining the handoff cost as a function of the available bandwidth and monetary cost. Roberts et al. (2007), actual RSS and bandwidth were chosen as two important parameters for the Waveform design. Niyato and Hossain (2008) propose a game theoretic frame work for radio resource management perform VHO in heterogeneous wireless networks. One main difficulty of the cost approach is its dependence on some parameters that are difficult to estimate, especially in large cellular networks. Barani and Fathy (2010) developed a cross-layer (Layer $2+3$ ) handoff management protocol CHMP, which calculates a dynamic value of the RSS threshold for handoff initiation by estimating MH's speed and predicting the handoff signaling delay of possible handoffs.

To sum up, the application scenario of current Vertical Handoff algorithms is relatively simple. For example, most Vertical Handoff algorithms only consider the pure Vertical Handoff scenario, where the algorithm only needs to decide when to use a $3 \mathrm{G}$ network and when to use a WLAN In fact, at any moment, there may be many available networks (homogeneous or heterogeneous) and the Handoff algorithm has to select the optimal network for Horizontal Handoff or Vertical Handoff from all the available candidates. For example, if the current access network of Mobile Host is a WLAN, the Mobile Assisted Handoff may sense many other WLANs and a 3G network at a particular moment and it has to decide whether to trigger Horizontal Handoff or Vertical Handoff. If the Horizontal Handoff trigger is selected, Mobile assisted handoff then needs to decide which WLAN is the optimal one (Yuan-Wu and $\mathrm{Li}, 2008$; Almudevar, 2008). Consequently, an analytical framework to evaluate VHO algorithms is needed to provide guidelines for optimization of handoff in heterogeneous wireless networks. It is also necessary to build reasonable and typical simulation models to evaluate the performance of $\mathrm{VHO}$ algorithms. The proposed work will provide an optimal solution to choose the network based on the type of service that is being carried out. As the work mainly concentrates on the application oriented approach the type of network and handover selection is also based on requirement It also concentrates on the identification of the source and destination and by locating them the path is chosen.

Solutions for mobility management: As today's requirement mainly focuses on voluminous information in real time which includes audio and video files where the continuity in the transmission path has to be maintained to give a seamless flow of information. Therefore, to provide a seamless flow of information in the heterogeneous environment, Mobility Management is considered as a major role. In the proposed study a generic tree is dynamically created from the geographical map generated between the source and the destination (as shown in Fig. 1) and also updations are made quite in advance so that as the device in mobility crosses the network the controls are just being communicated and processed in the background and the real communication takes place without any interruption.

As the metrics for mobility initially the status of the PDA (Mobility device) is being identified:

- Source stable Destination dynamic

- Source dynamic Destination stable

- Source dynamic Destination dynamic

- Source stable Destination stable

Note: The dynamic speed for the PDA is considered as the vehicular speed $(250 \mathrm{Kms} \mathrm{h})$

Location tracking: In this case the fourth state is considered as the source stable and destination stable and the shortest path is identified based on the signal strength and also the feasibility with minimum handover.

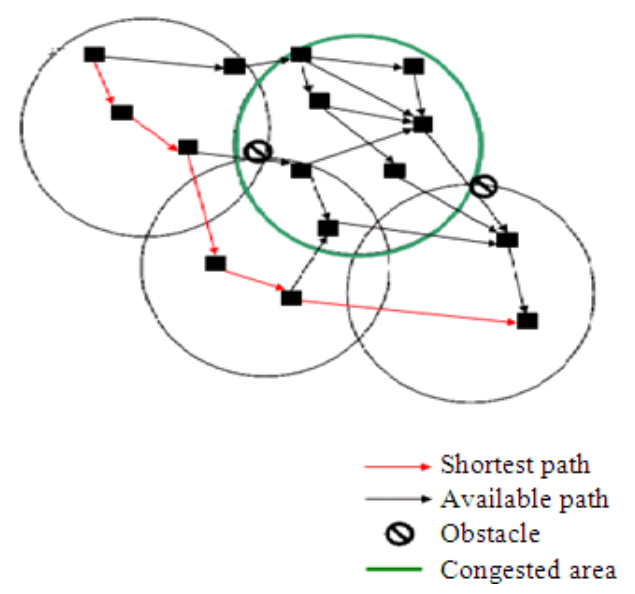

Fig. 1: Location tracking using the all pair shortest path algorithm 
In this method two classes that solve the all-pairs shortest-paths problem is being considered as a base. The first method is to run Dijkstra's algorithm from each vertex to get the shortest paths from that vertex to each of the others. If the priority queue with a heap is implemented, the worst-case running time for this approach is proportional to $\mathrm{VE} \lg \mathrm{V}$ and can improve this bound to VE for many types of networks by using a d-ary heap. The second method, which allows to solve the problem directly in time proportional to $\mathrm{V}^{3}$, is an extension of Warshall's algorithm that is known as Floyd's algorithm. The goal of the algorithms in this study is to support constant-time implementations of the query methods. Both of the algorithms that we consider use space proportional to $\mathrm{V}^{2}$ for the private data fields. The primary disadvantage of the general approach for a huge network is overcome by generating the graph dynamically by fixing the boundary of the source and the destination.

All-pairs shortest-paths by fixing the boundaries: The solutions to the all-pairs shortest-paths problem are all classes with a constructor and two query methods: a dist method that returns the length of the shortest path from the first given vertex to the second; and one of two possible path methods, either path, which returns a reference to the first edge on the shortest path, or pathR, which returns a reference to the final edge on the shortest path. If there is no such path, the path method returns 0 and dist is undefined. We use path or pathR as convenient for the algorithm under scrutiny; in practice, we might need to settle upon one or the other (or both) in the interface and use various transfer functions in implementations:

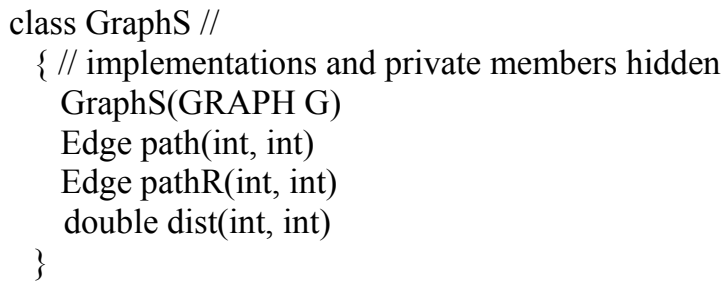

The first all-pairs shortest-paths ADT method implementation that we consider solves the problem by using Dijkstra's algorithm to solve the single-source problem for each vertex. We build an array of GraphSPT objects, one to solve the single-source problem for each vertex. This method generalizes the BFS-based method for unweighted undirected graphs. It is also similar to our use of a DFS that starts at each vertex to compute the transitive closure of unweighted digraphs.
Computing the diameter of a network: This client illustrates the use of the interface in It finds the longest of the shortest paths in the given network, prints the path and returns its weight (the diameter of the network):

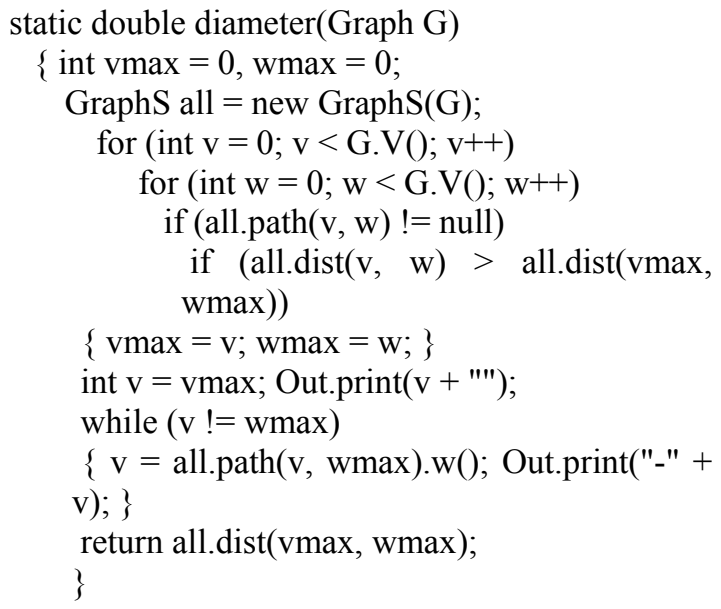

With Dijkstra's algorithm, we can find all shortest paths in a network that has nonnegative weights in time proportional to $\mathrm{VE} \log _{\mathrm{d}} \mathrm{V}$, where $\mathrm{d}=2$ if $\mathrm{E}<2 \mathrm{~V}$ and $\mathrm{d}$ $=\mathrm{E} / \mathrm{V}$ otherwise. For dense graphs, we could use an adjacency-matrix representation and avoid computing the reverse graph by implicitly transposing the matrix (interchanging the row and column indices), Developing an implementation along these lines is an interesting programming exercise and leads to a compact implementation; however, a different approach, which we consider next, admits an even more compact implementation.

Algorithm for all shortest paths for dynamic source: This class uses Dijkstra's algorithm to build an SPT for each vertex so that it can answer pathR anddist queries for any pair of vertices:

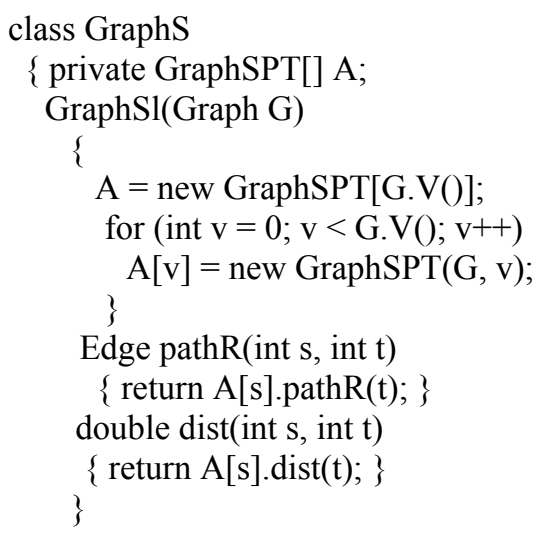


The method of choice for solving the all-pairs shortest-paths problem in dense graphs, was same as previous method, except that instead of using the logical or operation to keep track of the existence of paths, it checks distances for each edge to determine whether that edge is part of a new shorter path.

Algorithm for all shortest paths for dynamic source and destination: After initializing the distances and paths matrices with the graph's edges, we do a series of relaxation operations to compute the shortest paths. The algorithm is simple to implement, but verifying that it computes the shortest paths is more complicated:

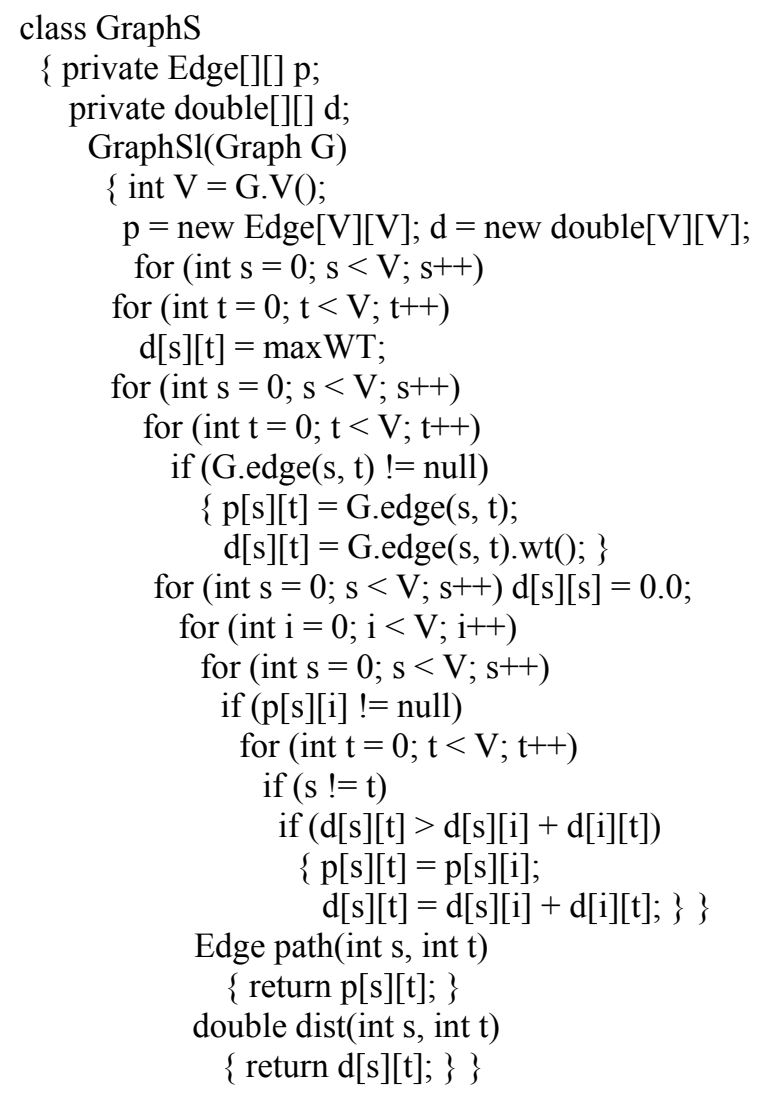

With this algorithm, we can find all shortest paths in a network in time proportional to $\mathrm{V}^{3}$.

Proof: The running time is immediate from inspection of the code. The ith iteration of the loop computes a shortest path from $s$ to $t$ in the network that does not include any vertices with indices greater than $\mathrm{i}$ (except possibly the endpoints $s$ and $t$ ). Assuming this fact to be true for the ith iteration of the loop, we prove it to be true for the $(i+1)$ st iteration of the loop. A shortest path from $s$ to $t$ that does not include any vertices with indices greater than $\mathrm{i}+1$ is either (i) a path from $s$ to $t$ that does not include any vertices with indices greater than $\mathrm{i}$, of length $\mathrm{d}[\mathrm{s}][\mathrm{t}]$, that was found on a previous iteration of the loop, by the inductive hypothesis; or (ii) comprising a path from $\mathrm{s}$ to $\mathrm{i}$ and a path from $i$ to $t$, neither of which includes any vertices with indices greater than $i$, in which case the inner loop sets $d[s][t]$. An entry in the distance matrix has the length of the shortest known path connecting the vertices corresponding to the given row and column; the corresponding entry in the paths matrix gives the next vertex on that path.

Session management: The Session Management Protocol (SMP) lies in the application layer and the major study is to identify the required resources based on the type of data/information that has to be transmitted / shared. Generally the data/ information can be categorized based on their application and the usage. The different types of information types are given in Fig. 4. Based on the application of the data/information the session management protocol is activated and the type of network is also selected. If the application involves huge data transmission then the bandwidth is shared and the network is chosen appropriately. The main goal is to provide a seamless flow of information without connectivity failure as the terminals are in mobile.

To support IP QoS, the Internet Engineering Task Force (IETF) recommends integrated services (IntServ) and differentiated services (DiffServ) (Olama et al., 2008). These services also are expected to be effective in all-IP-based 4G networks. Since 4G networks will support multimedia traffic, we must visit the issue of providing IP QoS in IP based wireless access networks (Olama et al., 2008) and propose ITRAS for QoS support in 4G networks, where the decision of radio resource allocation follows IntServ or DiffServ policy.

IntServ uses Resource Reservation Protocol (RSVP) to reserve bandwidth during the session setup. As a first step in RSVP, the source sends a QoS request message of PATH to the receiver through intermediate routers that run an admission and a policy control. If the sender receives RESV returned from the receiver through the reverse route as an indication of QoS guarantee, it initiates the session. If each router along the path receives packets, it classifies and schedules them. IntServ ensures strict QoS, but each router must implement RSVP and maintain per-flow state, which can cause difficulties in a large-scale network. DiffServ, on the other hand, does not require a signaling protocol and cooperation among nodes. As the QoS level of a packet is indicated by the DS field of 
IP header (TOS (type of service) field in IPv4, Traffic Class field in IPv6), each domain can deal with it independently. After the packet is classified, each router can mark, shape, or drop it according to network status. Since DiffServ is not so rigorous as IntServ, it is scalable in supporting QoS statistically.

Proposed service method: Given the requirements for seamless mobility listed above, the roles to be supported by a mobility management function exploiting the terminal capability to access several radio access networks are the following:

- Selection of the access network at application launch. This role is ensured by mobility management subfunctions here referred to as serviceto-radio mapping control

- Triggering of the handover during a session. The mobility management function aims at always providing the best access network to the terminal

- A terminal-centric selection without network assistance or recommendation

- A network-controlled selection within network entities, based on both terminal and access network measurements, enforcing decisions on the terminal

- Network-assisted selection on the terminal side, the network providing operator policies and access/core load information (joint terminal/network decisions). When only one access remains available, network-assisted selection is applied; when access selection is triggered by network load considerations, network control may be used for load balancing

- Finally, for access network selection, the mobility management function must retrieve the status of resource usage in each access network. This information is provided by an "access network resource management" function, which computes a technology-independent abstracted view of access resource availability

Functional entities involved in mobility management: The mobility management functions are supported by functional entities described below that are responsible for selecting the best access network for each terminal. They may be triggered at application launch or during the time of connection establishment:

- Generate a geographical map between the source and the destination based on the mode of transport

- Tabulate the list of service providers with their frequency and coverage
- Create an acyclic graph by pointing out the service providers and the geographical scenarios

- Form a topological graph from the acyclic graph by removing the obstacles and considering the signal strength

- Get the traffic status of each and every link from source to destination

- Now create a path from source to destination based on the network traffic and also by choosing the shortest path with minimum handovers

- For all the network in the specified path between the source and destination

- Get the bandwidth of the network

- Calculate the average traffic of the network

- Note down the obstacles on the way

- Note down the active service providers with their coverage area

- Note down the type of network services [3G, $3 \mathrm{GPP}]$

- Calculate the traffic density in the in the network

$\mathrm{NLc}+\Delta \mathrm{E} \leq \mathrm{NLth} \rightarrow$ take the same path

$\mathrm{NLc} \rightarrow$ Current Network Load

$\Delta \mathrm{E} \rightarrow$ Estimated Increase in Load

NLth $\rightarrow$ Network Threshold Load

- Generate a shortest path based on

- The maximum signal coverage

- Traffic density below the NLth

- Continue the transmission in the specified path

- Continue the same procedure till the source reaches the destination or the source is stable or not in mobility

Assumptions made for the analysis: Atleast one type of service provider is available within the limit

\section{RESULTS}

The results to provide the seamless flow of information in a practical context that addresses the integrating of cellular and WLAN access networks are considered. In order to implement the different functions listed earlier, some initial technological choices need to be made. First, only intertechnology handoffs WLAN are considered in the seamless mobility architecture. Intratechnology handoffs are taken care of by technology-specific mechanisms. Then Mobile IP has been chosen as the L3 protocol for handoff execution in the proof of concept and is used on top of either IPv4 or IPv6 in order to provide session continuity during intertechnology handoff. A clear separation of handoff decision and execution processes allows any evolution of IP protocols to minimize new 
care-of address configuration and rerouting latencies, for instance, to replace baseline Mobile IP without modifying the proposed architecture.

AS the existing technology is considered the 3GPP/UMTS network has a constant coverage for a limited traffic. The maximum users allowed is $80-85$. Also as the usage spectrum is considered only $80-85 \%$ of the available spectrum is utilized efficiently. The result is shown in Fig. 3. During which no traffic can be transmitted or received (it corresponds to traffic interruptions on Fig. 3. Upon returning to normal operation, a peak of traffic is observed when the terminal transmitting a burst of packets that could not be transmitted during the scanning periods of 200-400 $\mathrm{ms}$. In order to avoid perceivable interruption of voice transmission, an adaptive buffer has been set up on the receiver side, which enables the network to cope with "silences" but results in slightly increased latency. This configuration could further be improved by breaking the scanning period into shorter ones in order to avoid latency increase. However, this configuration may lead to lower measurement precision, so an acceptable compromise must be reached. In any case, this scenario is not considered for wider-scale deployment, since the latency on the EDGE network leads to unacceptable VoIP quality.

Another goal of the test bed was to assess performance of mobility management in the WLAN environment. As an example, we considered handoff delay for a $60 \mathrm{~kb} / \mathrm{s}$ Real-Time Transmission Protocol streaming service, with handoff delay defined as the delay between the first Real-Time Transmission Protocol packet on the new interface and the last packet on the old interface. When network control is enabled, the decision to perform handover is taken on load criteria: the streaming starts on the WLAN interface where other terminals load the AP when the network load reaches a given threshold, mobility management entities trigger the handover. In both cases handoff delay was about $0.05 \mathrm{~ms}$, because of Mobile IP and Generic Packet Radio Service network latencies. The results of the data rate in Fig. 2 also gives a clear picture that it was mainly based on the nature of the application and also the stability of the network varies upon the nature of the functions of the hardware deployed.

The higher transmission latency experienced in the cellular access network can be observed in the graph provided (Fig. 4). On the transmit side, the transmission is performed with no silence period. On the receive side, handing over to the cellular network introduces more latency, results in a silence period the order of magnitude of which is equal to the latency difference between both networks. The use of an adaptive buffer at the receiver side makes it transparent to the user which is reflected as a smooth seamless flow in the heterogeneous Networks. When considering the 3G/ Wimax cellular network, the number of users is high compared with the other networks and also had a wider coverage but there is a pitfall at the end, the bandwidth fluctuates beyond $80 \%$. At the time of mobility the network coverage is limited as shown in Fig. 5.

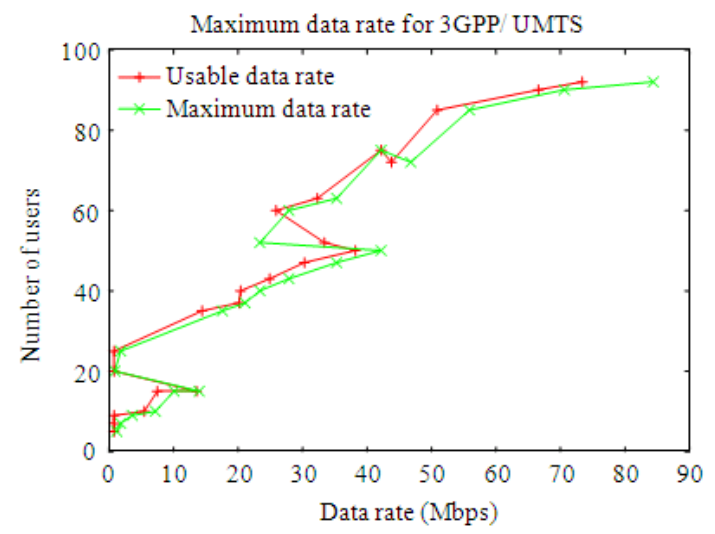

Fig. 2: Data rate of the network 3GPP/ UMTS

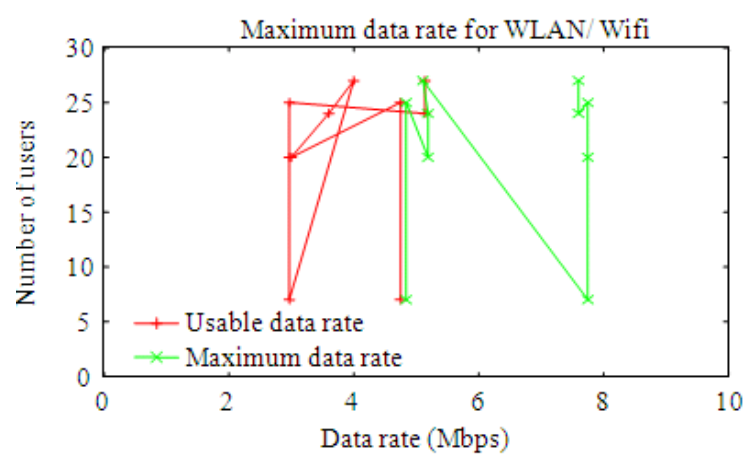

Fig. 3: Data Rate of WLAN Network

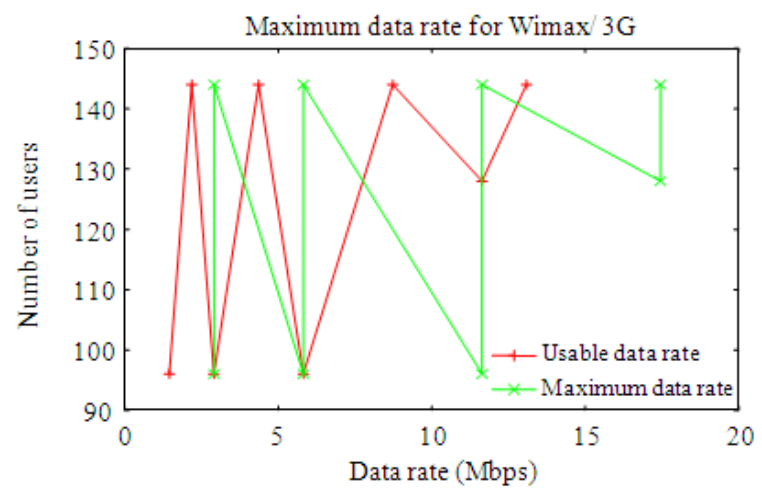

Fig. 4: Data rate in the $3 \mathrm{G}$ environment 


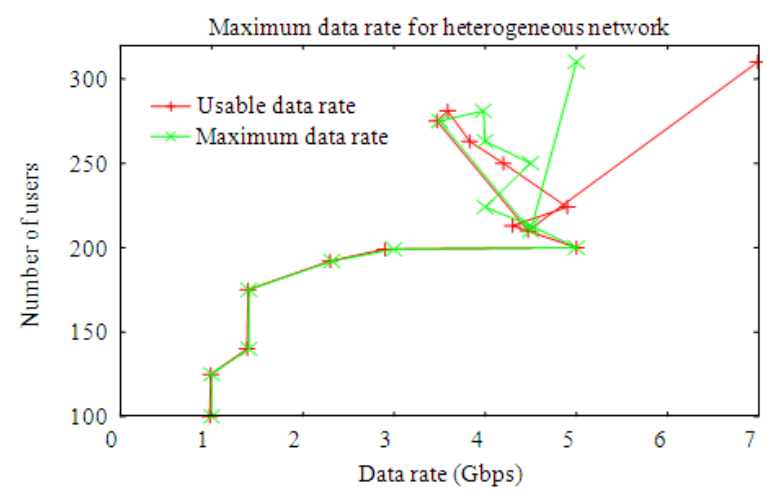

Fig. 5: Data rate in the heterogeneous network environment

By considering the positive measures of the above mentioned networks and by having a thorough understanding between the available networks the heterogeneous network is designed. The heterogeneous network provides maximum throughput, minimum number of handoffs and maximum coverage at mobile. By designing a proper QoS standard and having proper understanding between the network the desires which are explained at the initial paragraph can be achieved. By improving the performance measures by deploying and allocating the code cpectrum for the 3GPP network and by having proper power management in the $3 \mathrm{G}$ network and by making use of antennas with wider coverage in WLAN environment, the available bandwidth can be maximum utilized and also the number of handoffs can be reduced as the nature of the network present in the graphical architecture between the source and the destination is studied in advance, a maximum throughput can be achieved with minimum tolerable delay or no delay based on the nature of the information that is taken for transmission. The data rate of the heterogeneous network is very close to the available rate as shown in Fig. 5.

\section{CONCLUSION}

Results have confirmed the feasibility of the approach; its scalability to large geographical areas has to be confirmed with additional validation through simulations and trials. A possible stepwise approach to the deployment of the different functional elements of the presented architecture is defined. In this approach a vector based location tracking and management is only considered for the seamless flow. By combining the parameters such as signal strength and delay management in flow and also the formats of the information we can have a seamless flow. Also the QoS between the Radio Access Network's should be standardized in such a way that there is no mismatch of transmission from one type of environment to another type. Finally, with the advent of research on moving networks (e.g., Network Mobility), in which the whole network is mobile, the integration of WLANs and WMANs can improve mobile network connectivity. It is expected that public transportation (trains, buses, airplanes, ships) will popularize Internet access through WiFi connectivity to passengers while in movement. To this end, it will be equipped with a bridge to communicate with external networks such as WiMAX. Moreover, seamless mobility is still an issue as clients may be equipped with both interfaces and the vehicle gateway may also give support to $\mathrm{WiFi}$ external connectivity through dual gateways /interfaces (WiFi/ WiMAX) in order to offer fault tolerance and load balance between networks as well as new connectivity opportunities to passengers. Apart from serving the movement network, the mobile gateway can also be used by external clients, such as those outside the WiFi AP and WiMAX BS coverage areas, but that have the opportunity to download data or attain Internet access through the dual gateway belonging to the Vehicular Area Network (VAN).

\section{REFERENCES}

Almudevar, A., 2008. Approximate calibration-free trajectory reconstruction in a wireless network. IEEE Trans. Signal Process., 56: 3081-3088. DOI: 10.1109/TSP.2008.919090

Barani, H. and M. Fathy, 2010. An algorithm for localization in vehicular ad-hoc networks. J. Comput. Sci., 6: 168-172. DOI: 10.3844/jcssp.2010.168.172

ParveenSultana, H., M. Pounambal and D.P.V. Krishna, 2010. A fast handover scheme for multicasting in IPv6 based mobile ad hoc networks. J. Comput. Sci., 7: 90-94. DOI: 10.3844/jcssp.2011.90.94

Hassan, R., J. Irvine and I. Glove, 2009. Design and analysis of virtual bus transport using synchronous digital hierarchy/synchronous optical networking. J. Comput. Sci., 4: 1003-1011. DOI: 10.3844/jcssp.2008.1003.1011

Holis, J. and P. Pechac, 2008. Elevation dependent shadowing model for mobile communications via high altitude platforms in built-up areas. IEEE Trans. Antennas Propagation, 56: 1078-1084. DOI: 10.1109/TAP.2008.919209

Imre, S., 2007. Dynamic call admission control for uplink in 3G/4G CDMA-based systems. IEEE Trans. Vehicular Technol., 56: 2617-2629. DOI: 10.1109/TVT.2007.899932 
Ma, W., Y. Fang and P. Lin, 2007. Mobility management strategy based on user mobility patterns in wireless networks. IEEE Trans. Vehicular Technol., 56: 322-330. DOI: 10.1109/TVT.2006.883743

Niyato, D. and E. Hossain, 2008. A noncooperative game-theoretic framework for radio resource management in $4 \mathrm{G}$ heterogeneous wireless access networks. IEEE Trans. Mobile Comput., 7: 332345. DOI: 10.1109/TMC.2007.70727

Olama, M.M., S.M. Djouadi, I.G. Papageorgiou and C.D. Charalambous, 2008. Position and velocity tracking in mobile networks using particle and kalman filtering with comparison. IEEE Trans. Vehicular Technol., 57: 1001-1010. DOI: 10.1109/TVT.2007.906370

Quintero, A. and E.D. Frutos, 2009. MPLS based architecture for mobility and end-to-end QoS support in fourth generation mobile networks. J. Comput. Sci., 5: 255-262. DOI: 10.3844/jcssp.2009.255.262

Roberts, M.L., M.A. Temple, R.A. Raines, R.F. Mills and M.E. Oxley, 2007. Communication waveform design using an adaptive Spectrally Modulated, Spectrally Encoded (SMSE) framework. IEEE J. Selected Topics Signal Process., 1: 203-213. DOI: 10.1109/JSTSP.2007.897061
Rodriguez-Dagnino, R.M. and H. Takagi, 2007. Movement-based location management for general cell residence times in wireless networks. IEEE Trans. Vehicular Technol., 56: 2713-2722. DOI: 10.1109/TVT.2007.900377

Tamilselvan, G.M. and A. Shanmugam, 2011. Interference mitigation in IEEE 802.15.4-A cluster based scheduling approach. J. Comput. Sci., 7: 8089. DOI: 10.3844 jessp.2011.80.89

Yu, F. and V. Krishnamurthy, 2007. Optimal joint session admission control in integrated WLAN and CDMA cellular networks with vertical handoff. IEEE Trans. Mobile Comput., 6: 126-139. DOI: 10.1109/TMC.2007.250676

Yuan- $\mathrm{Wu}, \mathrm{Y}$. and $\mathrm{Y}$. Li, 2008. Iterative and diversity techniques for uplink MC-CDMA mobile systems with full load. IEEE Trans. Vehicular Technol., 57: 1040-1048. DOI: 10.1109/TVT.2007.906373

Zhu, Y.-H. and V.C.M. Leung, 2007. Optimization of sequential paging in movement-based location management based on movement statistics. IEEE Trans. Vehicular Technol., 56: 955-964. DOI: 10.1109/TVT.2007.891403 